\title{
On evaluation of ensemble precipitation forecasts with observation-based ensembles
}

\author{
B. Ahrens ${ }^{1}$ and S. Jaun ${ }^{2}$ \\ ${ }^{1}$ Institute for Atmosphere and Environment, University of Frankfurt, Germany \\ ${ }^{2}$ Institute for Atmospheric and Climate Science, ETH Zurich, Switzerland
}

Received: 4 August 2006 - Revised: 5 January 2007 - Accepted: 19 January 2007 - Published: 26 April 2007

\begin{abstract}
Spatial interpolation of precipitation data is uncertain. How important is this uncertainty and how can it be considered in evaluation of high-resolution probabilistic precipitation forecasts? These questions are discussed by experimental evaluation of the COSMO consortium's limitedarea ensemble prediction system COSMO-LEPS. The applied performance measure is the often used Brier skill score (BSS). The observational references in the evaluation are (a) analyzed rain gauge data by ordinary Kriging and (b) ensembles of interpolated rain gauge data by stochastic simulation. This permits the consideration of either a deterministic reference (the event is observed or not with $100 \%$ certainty) or a probabilistic reference that makes allowance for uncertainties in spatial averaging. The evaluation experiments show that the evaluation uncertainties are substantial even for the large area $\left(41300 \mathrm{~km}^{2}\right)$ of Switzerland with a mean rain gauge distance as good as $7 \mathrm{~km}$ : the one- to three-day precipitation forecasts have skill decreasing with forecast lead time but the one- and two-day forecast performances differ not significantly.
\end{abstract}

\section{Introduction}

Weather forecast systems have to be evaluated. Nowadays, limited-area numerical weather prediction models provide meteorological forecasts with kilometer-scale horizontal grid spacing. High-resolution precipitation forecasts are of primary interest. For example, in flood forecasting systems the precipitation details are a crucial input parameter.

A typical distance between precipitation observation sites with daily observation frequency in the European Alps is $10 \mathrm{~km}$ (cf. Fig. 1 for the distribution of precipitation stations in Switzerland). This is a comparatively dense observation

Correspondence to: B. Ahrens

(bodo.ahrens@iau.uni-frankfurt.de) network but precipitation is a quantity with high spatial variability. Therefore, it is a valid question to ask if such a density of observations allows for evaluation of daily precipitation forecasts in mountainous catchments with a typical area as small as about $1500 \mathrm{~km}^{2}$ ?

Recently, ensemble prediction systems (EPS) became operational which predict forecast probabilities by integration of an ensemble of numerical weather prediction models from slightly different initial states and model parameters (Ehrendorfer, 1997; Palmer, 2000). The motivation for the EPS is that the spread in the ensemble forecasts indicates forecast uncertainty and the interpretation of the forecast probabilities provides better results than interpretation of one single deterministic forecast that is initiated by the best known but nethertheless uncertain atmospheric state. Zhu et al. (2002) showed with a simple cost-loss model that for most users the ensemble forecasts offer a higher economic value than the deterministic forecast.

Here, EPS precipitation forecasts of the limited-area EPS COSMO-LEPS (Montani et al., 2003) with grid-spacing of $10 \mathrm{~km}$ are evaluated for the year 2005 for Switzerland and for three selected catchments (cf. Fig. 1). These three catchments are one pre-alpine catchment, the Thur, and two alpine catchments, the Aare (part of an elongated wet anomaly extending along the northern rim of the Alps) and the Hinterrhein (relatively dry inner-alpine area).

For the evaluation exercise presented here, we apply the commonly used Brier skill score (BSS). The BSS assess the probability forecasts of dichotomous events (e.g. the probability of more than $10 \mathrm{~mm}$ precipitation in the period and area of interest). In BSS application the observational reference is typically assumed to be certain: the observed event probability is either zero or one. The uncertainty in the observed catchment precipitation is often neglected.

This and the advantage of generating ensembles of interpolated observational fields, i.e. a probabilistic reference, through stochastic simulations is discussed in Sect. 4. Before

Published by Copernicus GmbH on behalf of the European Geosciences Union. 


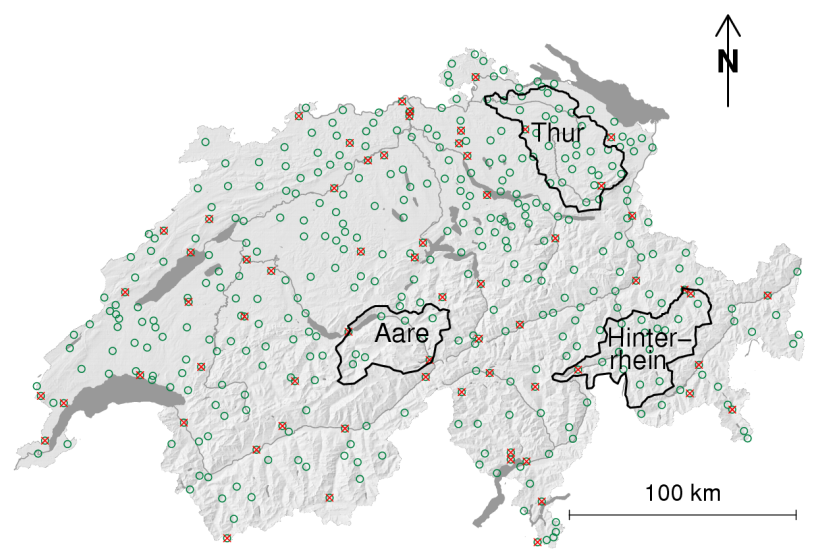

Fig. 1. Switzerland (total area: $41300 \mathrm{~km}^{2}$ ) and three catchments named Thur $\left(1700 \mathrm{~km}^{2}\right)$, Aare $\left(1200 \mathrm{~km}^{2}\right)$, and Hinterrhein $\left(1500 \mathrm{~km}^{2}\right)$. The circles show the locations of the rain station network ALL and the subset indicated by the red crosses show the locations of the network SUB.

that we introduce the available observational data and the evaluated limited-area EPS. Finally, evaluation results with and without observation uncertainty will be discussed in Sect. 5 and some concluding remarks will be given.

\section{Precipitation data}

This paper investigates precipitation in Switzerland and smaller catchments in the year 2005. The considered temporal resolution of the evaluation is daily. The reference are precipitation data as observed by the Swiss conventional precipitation station network available through the national weather service MeteoSwiss with about 430 stations in 2005 and a mean next-neighbor distance of about $7 \mathrm{~km}$. The data from this dense network is named ALL here. Also considered in the evaluation is a coarser data subset observed by 65 stations, which are located close to stations of the automatic measurement network ANETZ of MeteoSwiss with mean next-neighbor station distance of about $17 \mathrm{~km}$. This subset resembles the data availability in case of near realtime evaluation or in less densely observed regions and is named SUB. ANETZ data itself is not applied to avoid problems with mixing of different station types in the evaluation.

The spatial distributions of the two station sets are illustrated in Fig. 1. Within the considered catchments the numbers of stations are of the order of ten in case of ALL but only of two in case of SUB. Therefore, differences in evaluation with the different data sets are to be expected.

\section{Limited-area prediction system COSMO-LEPS}

The experimentally evaluated ensemble data are forecast by the consortium for small-scale modeling limited-area ensem- ble prediction system COSMO-LEPS (Montani et al., 2003, and http://www.cosmo-model.org). The COSMO-LEPS implementation is formally validated in Marsigli et al. (2005). We selected the year 2005 as our evaluation period, since it has been without major changes in the operational LEPS setup. In that period the ensemble size was set to ten and each ensemble member's forecast with grid-spacing of $10 \mathrm{~km}$ was initiated each day at 12:00 UTC. Here precipitation simulations for the forecast hours 18 to $42 \mathrm{~h}, 42$ to $66 \mathrm{~h}$, and 66 to $90 \mathrm{~h}$ (the one-, two-, and three-day forecasts, respectively) are assessed.

Each LEPS member is nested into a different representative forecast of a coarser-grid global EPS (the operational ensemble forecast of the European Centre of Medium Range Forecasts, Reading). These representative global members are selected by grouping the global members into ten clusters based on the analysis of wind and vorticity fields over a domain covering most of Europe (Molteni et al., 2001). From each cluster the central member (with minimum distance to all cluster members) is chosen to host a limited-area forecast. In the evaluation presented below we consider limited-area EPS members either weighted with cluster size or not.

Figure 2 shows the one-day forecast of the LEPS member that is driven by the most representative member (the central member from the cluster with about $25 \%$ of the global members) for 21 August 2005. This precipitation event led to major flooding in the northern European Alps. Also given in Fig. 2 are interpolated precipitation observations (cf. next section). The forecast depicts the coarse-scale features of the precipitation pattern but also over-estimates precipitation substantially in the central region of the event.

\section{Evaluation method}

The direct model output at grid-box scale should not be applied and some temporal and spatial smoothing of the output is recommended for being numerically representative (e.g. Grasso, 2000; Ahrens, 2003). Here, daily catchment means of precipitation are evaluated with averages over at least 15 model grid-boxes and thus the forecasts can be assumed representative. But how to estimate representative observational references from the limited number of rain gauge stations available? This has to be done by interpolation and averaging to the catchment scale.

Here, we apply ordinary Kriging with a spherical variogram model as one interpolation method. Kriging variants are often proposed and applied in precipitation analysis (Creutin and Obled, 1982; Atkinson and Lloyd, 1998; Beck and Ahrens, 2004). For the necessary variogram estimation we adopted a sub-optimal but robust approach (Ahrens and Beck, 2006). From the daily data of the year 2005 we estimated from standardized observations a climatological variogram range to about $40 \mathrm{~km}$ with a sill of $1(\mathrm{~mm} / \mathrm{d})^{2}$ (by construction). For daily analyses the sill is rescaled with the data 

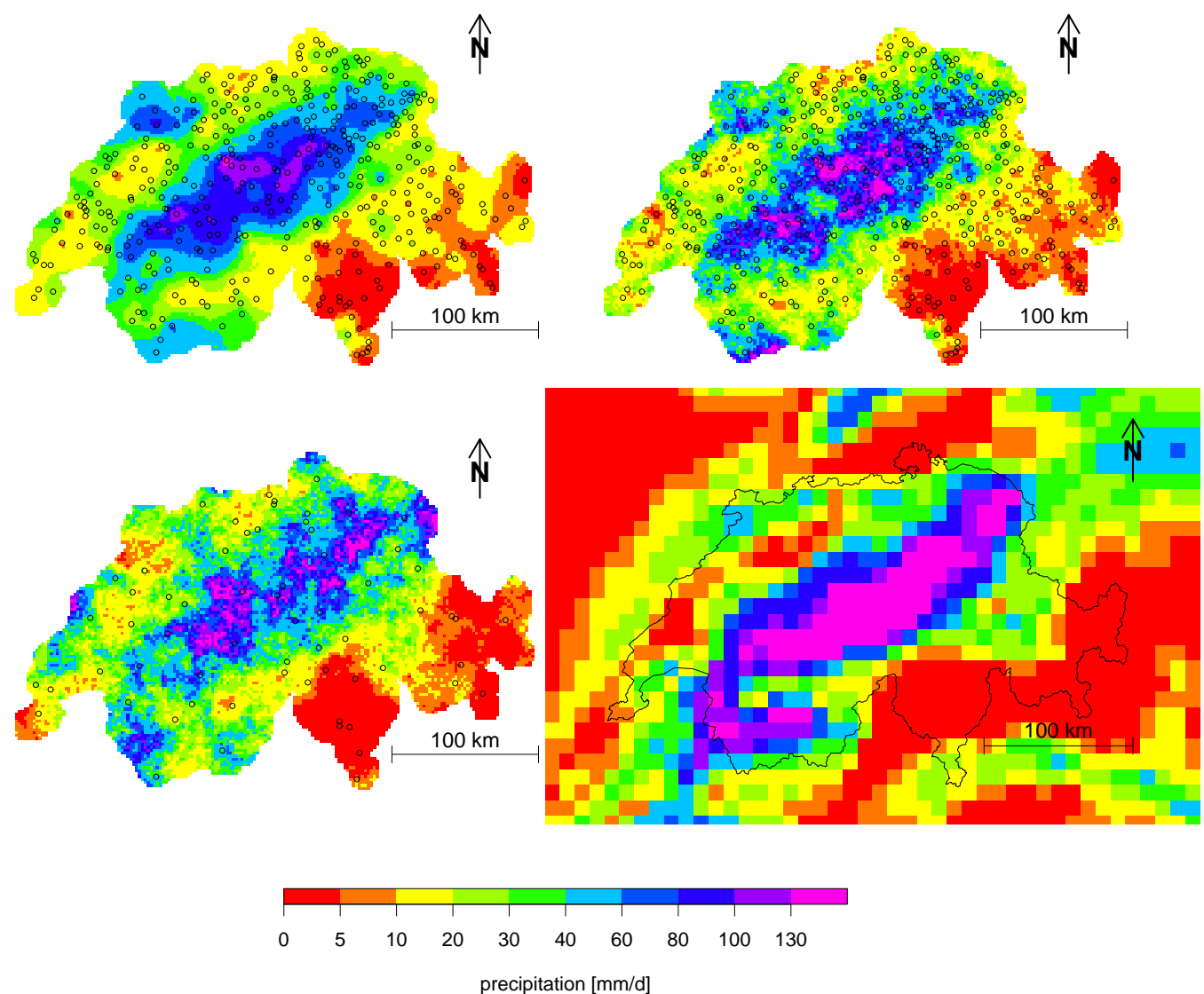

Fig. 2. Precipitation of 21 August 2005 in Switzerland as interpolated by Kriging (upper left panel), by stochastic simulation with ALL stations (upper right) or SUB stations (lower left), and as predicted by a 1-day forecast of the most representative COSMO-LEPS member (lower right). The locations of the considered stations are indicated by small circles.

variance. For either data set, ALL and SUB, a local neighborhood of 8 stations is considered in interpolation. Figure 2 shows the Kriging analysis for the day 21 August 2005 with ALL data.

Kriging is an example of a data-fitting technique. Therefore, it is expected that the interpolated fields underestimate the true field variance (the smoothing relationship of Kriging states that the interpolated field variance at any location is the data variance minus the Kriging variance) with the consequence that the variance of the catchment time series is underestimated. More important in evaluation is that the estimation of the interpolation errors is extremely difficult in case of precipitation since the stationarity and normality assumptions of Kriging are not very well fulfilled. Here, the areal precipitation estimate through ordinary Kriging is considered a deterministic observational reference (DOR) because no uncertainty in interpolation is considered.

An alternative interpolation approach is based on stochastic simulation of an ensemble of precipitation fields with conditioning on the available station data. The idea is to simulate stochastically field realizations that "honor" the observed data, their point values, their areal mean, and their covariance structure (Journel, 1974; Chilès, 1999; Ahrens and Beck, 2006). Therefore, the spatial variability is represented more realistically in the stochastic realizations than in Kriging. For the evaluation exercise a large ensemble of observation-based realizations is generated and thus a probabilistic observational reference (POR) is available. This allows the comparison of probabilistic EPS forecasts against the POR by comparison of probability distributions. Additionally, an ensemble of comparisons against the multiple realizations reference (MRR) of the observational ensemble can be performed and the spread in these comparisons provides a precision measure for the evaluation without troublesome estimation and interpretation of the Kriging variance. Averaging the ensemble of observation-based realizations yields a data-fitting technique (and this mean interpolator is thus smoother than any ensemble member) and is in the limit of large ensembles equivalent to a Kriging approach. The ensemble average yields another DOR in the following evaluation.

Stochastic interpolation is done by conditioned sequential Gaussian simulation (e.g., Johnson, 1987; Chilès, 1999, Chap. 7) as it is implemented in the geostatistical software 


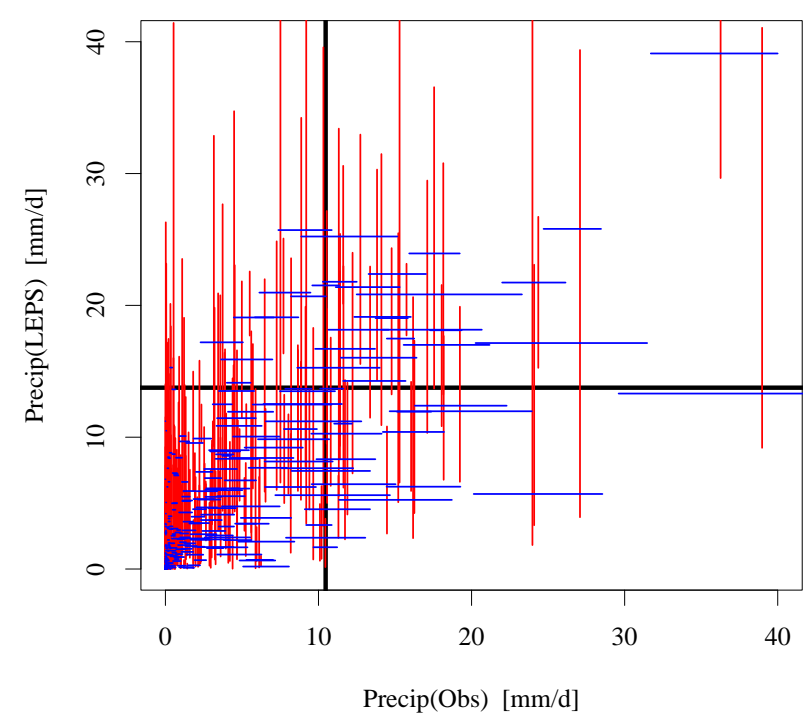

Fig. 3. The median LEPS 1-day forecasts versus the median observation-based stochastic interpolations of the SUB network. The precipitation values are daily and Swiss averages. The bars indicate the $90 \%$ confidence intervals of the forecasts (red) and of the interpolations (blue). The thick black lines give the 90th percentiles of the interpolations $(10.5 \mathrm{~mm} / \mathrm{d})$ and the forecasts $(13.8 \mathrm{~mm} / \mathrm{d})$.

package "gstat" (Pebesma, 2004). Sequential simulation involves the generation of a Gaussian random field, conditioned to the observed data, that honors the variogram of the random field. Since daily precipitation is a non-Gaussian, non-negative process, the data has been normalized by a logarithmic transformation and applying variogram estimates for the transformed data based on rescaling of the climatological variogram with an estimated climatological range of about $100 \mathrm{~km}$. For each day and data set an ensemble of realizations with one hundred members is generated and applied in the following comparisons. Each ensemble member is less accurate than the Kriging analysis in a squared-error sense by construction, but respects the covariance structure given by the observations.

Figure 2 shows two realizations of stochastic interpolation: one is conditioned on ALL and the other on SUB observations. As expected the stochastic interpolation is rougher than Kriging. Additionally, it can be seen that the conditioning by ALL is more restrictive than by SUB by comparison with the Kriging interpolation of the dense ALL network data. Figure 3 illustrates that in case of the SUB network there is even for daily and Swiss averages substantial scatter in the observational reference. The scatter is even larger for the smaller catchments (not shown).

Optimal interpolation of precipitation fields is an active field of research. The remaining deficiencies of the Kriging analysis and stochastic simulation upscaling motivate the discussion of the advantages of PORs over DORs. Neverthe- less, the applied methods are state-of-the-art for daily highresolution precipitation interpolation.

An often applied performance measure in evaluation of probabilistic forecasts also applied here is the Brier skill score, BSS, (cf. Stanski et al., 1989; Wilks, 2006, and references wherein). The BSS compares probability forecasts $Y_{t}=P\left(y_{t} \leq y_{0}\right)$ at dates $t=1,2, \ldots, T$ of forecast events $y_{t} \leq y_{0} \quad\left(y_{0}\right.$ is a chosen event threshold: e.g. $10 \mathrm{~mm} / \mathrm{d}$ in case of precipitation forecasts $y_{t}$ ) with the observed event probabilities $O_{t}=P\left(x_{t} \leq x_{0}\right)$ of some observational quantity $x_{t}$ with related threshold $x_{0}$. Commonly, the observations are assumed perfect and thus $O_{t} \in\{0,1\}$ - the event occurred or did not. This is the assumption made in evaluation with DOR. Figure 3 shows that our knowledge about observed event occurrence is uncertain: for several precipitation days the 90th percentile threshold is within the confidence interval of the reference values. Therefore, POR has to be applied and the $O_{t}$ 's codomain is the interval $[0,1]$.

The BSS is defined by

$\mathrm{BSS}=1-\frac{\mathrm{BS}(Y, O)}{\mathrm{BS}(C, O)}$

with the Brier score

$\mathrm{BS}(Y, O)=1 / T \sum_{t=1}^{T}\left(Y_{t}-O_{t}\right)^{2}$

The BS is essentially the mean squared error of the probabilistic forecast. The BS $(C, O)$ of some climatological forecast $C$ is introduced as a reference forecast in the BSS for normalization. The skill score equals one in case of perfect forecasts (a perfect forecast of an uncertain observational reference is uncertain itself) and zero if the evaluated forecast skill compares to the skill of the climatology.

The estimation of forecast probabilities from small EPS leads to biased BSS values (Müller et al., 2005). The COSMO-LEPS ensemble size is ten only. Therefore, we debiased the BSS following Weigel et al. (2006). The climatological probability of some precipitation forecast threshold can not be estimated reliably because of the short period of available COSMO-LEPS data. We applied instead the 90th percentiles in 2005 depending on the data-set (forecast or observation-based, catchment) as thresholds. For example, the threshold for the kriged reference with ALL data in Switzerland is $9.5 \mathrm{~mm} / \mathrm{d}$, for the stochastically interpolated realizations $9.7 \mathrm{~mm} / \mathrm{d}$, and for the EPS forecasts about $13.5 \mathrm{~mm} / \mathrm{d}$. This data-set dependent selection of thresholds is equivalent to some forecast post-processing and improves the BSS.

\section{Results}

As mentioned above the probabilistic EPS forecasts are usually compared against deterministic references (DORs). This 
Table 1. BSS values for the 1-,2-, and 3-day COSMO-LEPS forecasts with weighted members. Different observational references based on ALL observations are applied in the BSS estimation: (a) deterministic observational references (DOR) by Kriging/averaging of ensembles of stochastically interpolated fields, (b) multiple stochastic realizations (MRR) yielding an BSS ensemble (the first and third quartiles of the BSS distributions are given), and (c) probabilistic references (POR).

\begin{tabular}{lccc}
\hline & 1 day & 2 days & 3 days \\
\hline \multicolumn{4}{c}{ Switzerland } \\
DOR & $.44 / .45$ & $.42 / .45$ & $.36 / .33$ \\
MRR & $.44-.48$ & $.43-.45$ & $.31-.33$ \\
POR & .48 & .46 & .34 \\
& \multicolumn{4}{c}{ Thur } \\
DOR & $.44 / .46$ & $.36 / .37$ & $.27 / .28$ \\
MRR & $.39-.44$ & $.32-.37$ & $.24-.28$ \\
POR & .45 & .37 & .28 \\
& \multicolumn{4}{c}{ Hinterrhein } \\
DOR & $.20 / .18$ & $.18 / .17$ & $.19 / .17$ \\
MRR & $.18-.20$ & $.17-.18$ & $.17-.20$ \\
POR & .20 & .18 & .19 \\
& \multicolumn{4}{c}{ Aare } \\
DOR & $.37 / .36$ & $.29 / .20$ & $.25 / .21$ \\
MRR & $.31-.36$ & $.18-.22$ & $.21-.24$ \\
POR & .38 & .23 & .26 \\
\hline
\end{tabular}

paper generates DORs either by Kriging or by averaging ensembles of stochastic interpolations of the precipitation observations followed by averaging to the evaluation areas. For large ensembles applying the same variogram models etc. these references converge. Here, the observational ensemble consists of one hundred members and there are differences in the climatological variogram and data normalization. Table 1 shows that these differences yield differences in the BSS. It is interesting to note that the second method gives slightly better BSS in the larger and relatively better observed areas (Switzerland and Thur) and slightly worse results in the more difficult areas (Hinterrhein and Aare with relatively less observations, but also, as the smaller BSS values indicate, the more challenging forecast regions).

Table 1 also shows the inter-quartile range of BSS values if the probabilistic forecasts are compared against the stochastic MRRs. The spread is substantial and larger than the differences between the deterministic results. For example, taking the spread into account the performance of one- or two-day forecasts for Switzerland do not differ significantly (the inter-quartile ranges overlap) for the $10 \%$ heaviest rain events which are evaluated in this paper. The forecasts for the Hinterrhein are less good as for the other areas and there is no significant difference between one-, two-, and even threeday forecasts. In this area only rather large-scale rain events are well forecasted and those are well represented in the EPS quite independent of the forecast lead time (not shown).

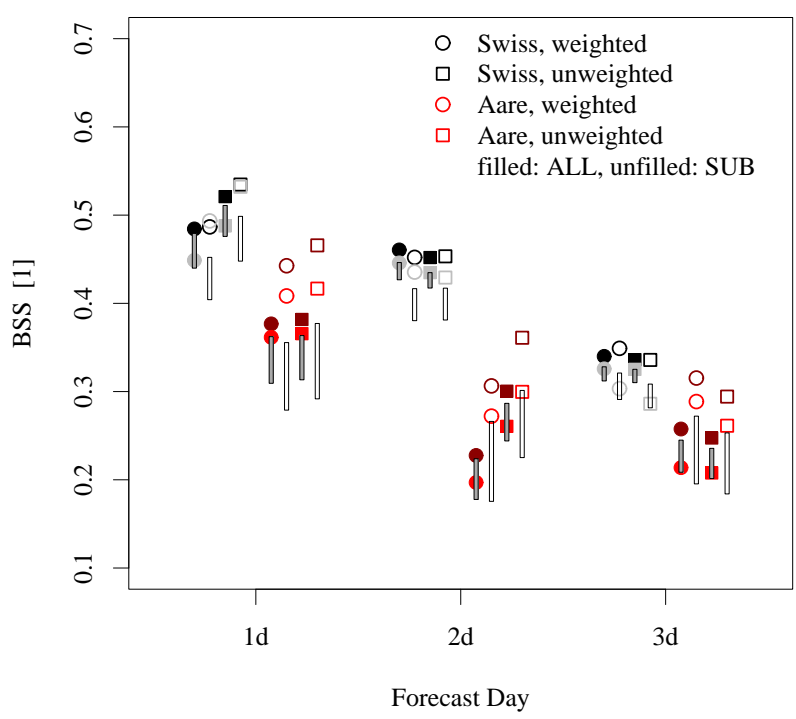

Fig. 4. BSSs of ensemble forecasts in the Swiss and Aare areas. The darker symbols (black, dark red) indicate evaluation against the probabilistic reference, the lighter symbols (grey, light red) indicate evaluation against the deterministic ensemble mean reference, the bars indicate the inter-quartile range of evaluation results against single members of the observational ensemble, and the filled and unfilled symbols and bars show the results for the networks ALL and SUB, respectively. Bullets and squares with the close-by bars discriminate between weighted and unweighted forecast ensembles, respectively.

Also given in the Table are the BSSs if the probabilistic forecasts are evaluated against PORs. These BSSs tend to be better than for the deterministic or single member evaluations. This is not surprising since in this case $O_{t}$ can take values between zero and one and - remembering that the BS is a quadratic difference - this leads to smaller BSs and larger BSSs as long as the observational uncertainty is smaller than the forecast uncertainty. This is a welcome feature since an uncertainty in the observational reference should not punish the forecasts.

Figure 4 clearly shows that the evaluation uncertainty is larger for the smaller Aare catchment than for Switzerland. At the same time the forecast performance is at least one day better in the larger area. Again the consideration of the reference uncertainty through the PORs increases the BSSs. The difference in probabilistic or deterministic reference evaluation is especially large, as expected, in the Aare catchment if the coarse observation network SUB is considered.

As mentioned in Sect. 3 the LEPS members can be weighted by the size of the global forecast clusters. This should lead to better forecasts in general but not necessarily for Switzerland since the clustering is based on large-scale weather patterns. Figure 4 indicates that the one-day forecast for Switzerland is insignificantly better with unweighted members and that for two- and three-day forecast weighting marginally improves the forecasts. In case of the smaller 
Aare area the weighting is detrimental for one- and two-day forecasts. Non-uniform weighting decreases the spread in the forecast ensembles (the inter-quartile ranges in the forecast ensemble are $2.8 \mathrm{~mm} / \mathrm{d}$ for the weighted and $2.9 \mathrm{~mm} / \mathrm{d}$ for the unweighted one-day forecasts in the year-long average) and thus yields more extreme $Y_{t} \mathrm{~s}$ and smaller BSS values. For longer lead times the evaluation areas are increasingly influenced by large-scale weather patterns leading to the global clusters and thus the optimal selection of the global representative members yields an increase in spread $(4.6 \mathrm{~mm} / \mathrm{d}$ with and $4.5 \mathrm{~mm} / \mathrm{d}$ without for three-day forecasts) and this favors weighting (although insignificantly because of the evaluation uncertainty).

\section{Conclusions}

It is common practice in evaluation of probabilistic areal precipitation forecasts that the observational reference is assumed perfect neglecting spatial interpolation errors. This paper shows that generating ensembles of stochastically interpolated fields conditioned on the available data is a simple method for consideration of interpolation uncertainty in the evaluation. These ensembles allow the determination of ensembles of comparisons if the forecast is compared against every single ensemble member. The spread in the comparison ensemble easily delivers an evaluation uncertainty. This is demonstrated by estimation of ensembles of Brier skill score (BSS) values in evaluation. Additionally, the observational ensembles can be considered as a probabilistic reference in a probabilistic evaluation. This yields higher BSS values than with single, deterministic reference evaluation, and this is fair since in doubt it should be assumed that the forecasts perform well. Therefore, we suggest application of a reference ensemble for (a) estimation of evaluation uncertainty and (b) the estimation of the potentially best performance of the forecast given the reference's uncertainty.

Additional sources of evaluation uncertainty have to be considered besides horizontal interpolation errors. In mountainous areas the vertically inhomogeneous distribution of stations can lead to systematic errors. Further, wind and evaporation loss of the rain gauges yields precipitation undercatch up to several ten percent. These error sources additionally illustrate how challenging the evaluation of precipitation forecasts in small areas is and will be with ever increasing resolution of forecasts and forecast applications.

Acknowledgements. Data are provided by MeteoSwiss, Zurich. S. J. acknowledges support through NCCR-Climate, funded by the Swiss National Science Foundation.

Edited by: S. C. Michaelides and E. Amitai

Reviewed by: anonymous referees

\section{References}

Ahrens, B.: Evaluation of precipitation forecasting with the limited area model ALADIN in an Alpine watershed, Meteorol. Z., 12,
245-255, 2003.

Ahrens, B. and Beck, A.: On upscaling of rain-gauge data for evaluating numerical weather forecasts, Meteorol. Atmos. Phys., in print, 2007

Atkinson, P. and Lloyd, C.: Mapping precipitation in Switzerland with ordinary and indicator Kriging, J. Geographic Information and Dec. Analysis, 2, 65-76, 1998.

Beck, A. and Ahrens, B.: Multiresolution evaluation of precipitation forecasts over the European Alps, Meteorol. Z., 13, 55-62, 2004.

Chilès, J.-P.: Geostatistics: modeling spatial uncertainty, John Wiley \& Sons, New York, 1999.

Creutin, J. and Obled, C.: Objective analyses and mapping techniques for rainfall fields: an objective comparison, Water Resour Res., 18, 413-431, 1982.

Ehrendorfer, M.: Predicting the uncertainty of numerical weather forecasts: a review, Meteorol. Z., 6, 147-183, 1997.

Grasso, L. D.: The differentiation between grid spacing and resolution and their application to numerical modeling, Bull. Amer. Meteorol. Soc., 81, 579-580, 2000.

Johnson, M.: Multivariate Statistical Simulation, Wiley, New York, 1987.

Journel, A.: Geostatistics for conditional simulation of ore bodies, Econom. Geol., 69, 673-687, 1974.

Marsigli, C., Boccanera, F., Montani, A., and Paccagnella, T.: The COSMO-LEPS mesoscale ensemble system: validation of the methodology and verification, Nonlin. Processes Geophys., 12, 527-536, 2005, http://www.nonlin-processes-geophys.net/12/527/2005/.

Molteni, F., Buizza, R., Marsigli, C., Montani, A., Nerozzi, F., and Paccagnella, T.: A strategy for high-resolution ensemble prediction. I: Definition of representative members and global-model experiments, Quart. J. Roy. Meteorol. Soc., 127, 2069-2094, 2001.

Montani, A., Capaldo, M., Cesari, D., Marsigli, C., Modigliani, U., Nerozzi, F., Paccagnella, T., Patruno, P., and Tibaldi, S.: Operational limited-area ensemble forecasts based on the Lokal Modell, ECMWF Newsletter, 98, 2-7, 2003.

Müller, W., Appenzeller, C., Doblas-Reyes, F., and Liniger, M.: A debiased ranked probability skill score to evaluate probabilistic ensemble forecasts with small ensemble sizes, J. Climate, 18, 1513-1523, 2005

Palmer, T. N.: Predicting uncertainty forecasts of weather and climate, Rep. Prog. Phys., 63, 71-116, 2000.

Pebesma, E.: Multivariable geostatistics in S: the gstat package, Comp. \& Geosci., 30, 683-691, 2004.

Stanski, H., Wilson, L., and Burrows, W.: Survey of common verification methods in meteorology, Tech. Rep. WMO/TD No. 358, WMO World Weather Watch, 1989.

Weigel, A., Liniger, M., and Appenzeller, C.: The discrete Brier and ranked probability skill scores, Mon. Wea. Rev., 135, 118-124, 2007.

Wilks, D. S.: Statistical Methods in the Atmospheric Sciences, Academic Press, San Diego, 2006.

Zhu, Y., Toth, Z., Wobus, R., Richardson, D., and Mylne, K.: The economic value of ensemble-based weather forecasts, Bull. Amer. Meteorol. Soc., 83, 73-83, 2002. 